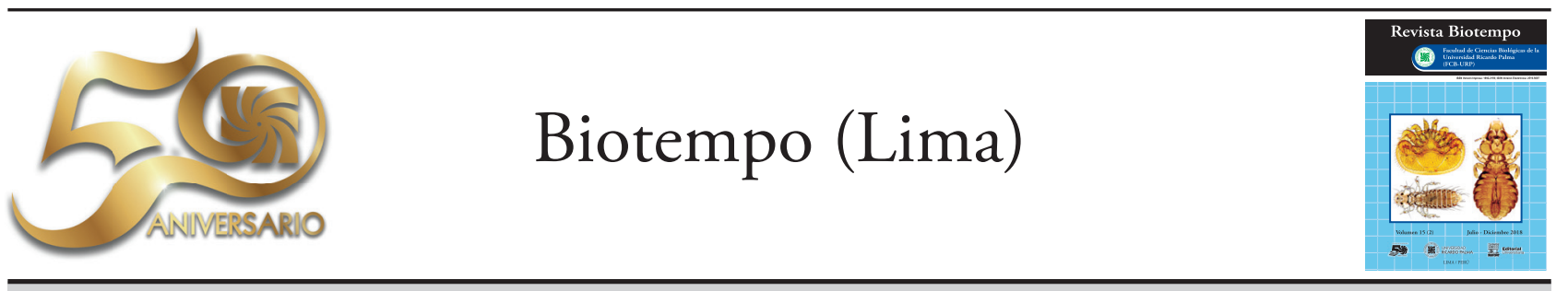

\author{
ORIGINAL ARTICLE / ARTÍCULO ORIGINAL
}

ECOTOXICOLOGY AS A PREDICTIVE TOOL ON THE SUSTAINABLE EVOLUTION OF AQUATIC SYSTEMS

\title{
ECOTOXICOLOGÍA COMO HERRAMIENTA PREDICTIVA EN LA EVOLUCIÓN SOSTENIBLE DE LOS SISTEMAS ACUÁTICOS
}

\author{
George Argota-Pérez ${ }^{1} \&$ José Iannacone ${ }^{2,3}$
}

1 Centro de Investigaciones Avanzadas y Formación Superior en Educación, Salud y Medio Ambiente "AMTAWI", Puno-Perú. george.argota@gmail.com

2 Laboratorio de Parasitología. Facultad de Ciencias Biológicas. Universidad Ricardo Palma (URP). Lima-Perú. joseiannacone@gmail.com

3 Laboratorio de Ecología y Biodiversidad Animal (LEBA). Facultad de Ciencias Naturales y Matemática. Universidad Nacional Federico Villarreal (UNFV). Lima-Perú

Author for correspondence: george.argota@gmail.com

\begin{abstract}
Pollution causes deterioration of the environmental components where certain branches of application know how to recognize the degree of equilibrium at the ecosystem level. The purpose of the study was to indicate ecotoxicology as a predictive tool on the sustainable evolution of aquatic systems. Through the analysis of content on theoretical aspects and practical uses referring to ecotoxicology, the environmental quality and sustainable evolution of the San Juan ecosystem of Santiago de Cuba, Cuba, was considered for 10 years (2008-2018). The variables of interest analyzed were the biological condition factor $(\mathrm{K})$ and the bioaccumulation of metals ( $\mathrm{Pb}$ and $\mathrm{Cd}$ ) in the gills (target organs) of the fish species Gambusia punctata (Poey, 1854) (Poeciliidae), which was selected in the biomonitor category. It was observed, statistically significant differences in the K indicator (for both sexes), which was measured every 5 years (2008, 2013 and 2018) where their values decreased (males / 2008: 0.45-0.47, 2013: 0 , 43-0.44, 2018: 0.37-0.41, females / 2008: $0.63-0.66,2013: 0.58-0.61,2018: 0.55-0.58)$ while that, there were no statistically significant differences in relation to the bioaccumulation of metals (considered as average values between males and females (ug. $\mathrm{g}^{-1}$ ), $\mathrm{Pb} / 2008$ : $0.04 \pm 0.01,2013: 0.03 \pm 0.02 ; 2018: 0.04 \pm 0.02 ; \mathrm{Cd} / 2008: 16.44 \pm 0.017 ; 2013: 16.77 \pm 0.011 ; 2018: 16.78 \pm$ 0.008). It was concluded that there was limited environmental stability over time and therefore; little sustainability as an ecosystem resource for San Juan, being recognized through the analysis of biomarkers which were based on the precepts of ecotoxicology.
\end{abstract}

Keywords: ecotoxicology - environmental - evolution - management instrument - risk prediction 


\section{RESUMEN}

La contaminación ocasiona deterioro de los componentes ambientales donde determinadas ramas del saber aplicativo permiten reconocer el grado de equilibrio a nivel de ecosistema. El propósito del estudio fue indicar la ecotoxicología como una herramienta predictiva sobre la evolución sostenible de los sistemas acuáticos. Mediante el análisis de contenido sobre aspectos teóricos y usos prácticos referido a la ecotoxicología se consideró, durante 10 años (20082018), la calidad ambiental y evolución sostenible del ecosistema San Juan de Santiago de Cuba, Cuba. Las variables de interés analizadas fueron el factor de condición biológico $(\mathrm{K})$ y la bioacumulación de metales $(\mathrm{Pb}$ y $\mathrm{Cd}$ ) en las branquias (órganos diana) de la especie de pez Gambusia punctata (Poey, 1854) (Poeciliidae), el cual fue seleccionado en categoría de biomonitor. Se observó, diferencias estadísticamente significativas en el indicador K (para ambos sexos), el cual se midió cada 5 ańos $(2008,2013$ y 2018) donde sus valores disminuyeron (machos / 2008: 0,45-0,47; 2013: 0,43-0,44; 2018: 0,37-0,41; hembras / 2008: 0,63-0,66; 2013: 0,58-0,61; 2018: 0,55-0,58) mientras que, no hubo diferencias estadísticamente significativas con relación a la bioacumulación de los metales (considerados como valores promedios entre machos y hembras (ug.g $\left.{ }^{-1}\right) ; \mathrm{Pb} / 2008: 0,04 \pm 0,01 ; 2013: 0,03 \pm 0,02 ; 2018: 0,04 \pm 0,02 ; \mathrm{Cd} / 2008$ : $16,44 \pm 0,017 ; 2013: 16,77 \pm 0,011 ; 2018: 16,78 \pm 0,008)$. Se concluyó que, existió limitada estabilidad ambiental en el tiempo y por ende; poca sostenibilidad como recurso ecosistémico para San Juan, siendo reconocido mediante el análisis de los biomarcadores los cuales se basaron en los preceptos de la ecotoxicología.

Palabras clave: ecotoxicología - evolución ambiental - intrumento de gestión - predicción de riesgo

\section{INTRODUCCIÓN}

Más de 600 sustancias activas (relevantes) se han registrado en todo el planeta (Weber et al., 2014), cuyas concentraciones promedio varían de acuerdo al tipo de molécula y al país de procedencia (Hughes et al., 2013); sin embargo, no existe duda sobre el mayor impacto que recepciona, la matriz ambiental del agua (Lopez et al., 2013) y por causa de las deficiencias en los diseños de las plantas de tratamientos convencionales, el desequilibrio se acrecienta (Pal et al., 2014). Entre las posibles consecuencias del desequilibrio ambiental de las aguas está la discontinuidad entre la interpretación de los daños que ocurre a nivel individual y de población (Forbes \& Galic, 2016) donde las respuestas biológicas para futuras investigaciones (Lam \& Gray, 2003; Stark et al., 2007) por lo general, suceden mediante el vínculo directo entre las fuentes contaminantes y el uso de modelos explícitamente probados a nivel individual (Simpson et al., 2013; O'Brien \& Keough, 2014). A pesar de esta relación, continúa siendo limitado, predecir el efecto sobre aquellos procesos que pudieran ocurrir por encima del nivel de población (Hommen et al., 2010; Pedersen et al., 2013).

Adicionalmente, pocos de los datos agrupados son utilizados en la toma de decisiones ante los riesgos ambientales presentados donde Destrieux et al. (2017) señalan que, la acumulación de datos, no solo debe aumentar el conocimiento de los posibles riesgos sino, permitir como instrumento la objetividad para contribuir a la evaluación rápida y eficiente lo que podría coadyuvar de igual modo, a la protección ambiental ante el impacto que se genera (Carter \& Howe, 2006; USEPA, 2015).

En múltiples encuentros científicos y/o políticos se ha referido que, los desequilibrios ambientales, no son tan simples de solucionar; pero, una pregunta surge ante tal preocupación: ¿si se desconoce los posibles efectos observados por no describirse umbrales naturales con relación a lo que se intenta producir entonces, por qué existen actualizaciones bajo ciertas incertidumbres?. $\mathrm{Al}$ parecer, la respuesta pudiera ser evidente, pues solo manifestarse con lógica racional en sí misma, es la solución. El agobio y la busca desesperada sobre qué hacer para transformar los desequilibrios ambientales, crea en numerosos profesionales, la facultad de pronosticar algún cambio hacia posible y adecuada mejora mediante la visión del entendimiento relacionada con la Ecotoxicología. En algún modo, pudiera ser totalmente cierto por su carácter preventivo y aplicativo, pero la tenencia de una cultura ambiental en la sociedad, significará la principal asignatura de aprendizaje y con ello, no mostrar una sumatoria de peligros generados.

Durante una reunión celebrada por el Comité del Consejo Internacional de Uniones Científicas con sede en la ciudad de Estocolmo-Suecia, el profesor e investigador francés Truhaut en 1969, sugirió por primera vez, la terminología Ecotoxicología donde hizo mención cómo, extensión natural de la Toxicología (ciencia que estudia los efectos de las sustancias tóxicas sobre los organismos individuales). Para el uso de esta terminología refirió indicación a dos efectos ecológicos ocasionados por 
los contaminantes: $1^{\text {ro }}$ ), la toxicidad hacia cualquier organismo; y $2^{\mathrm{do}}$ ), las alteraciones del medio en el cuál viven los organismos. La figura 1 muestra lo seńalado por Truhaut cuando indicó que, un contaminante al provocar la muerte sobre el $50 \%$ en los individuos dentro de una población podría tener poca significación pero, si un contaminante no provoca la muerte, aunque retarda una función biológica (efecto biológico no deseado) o altera el medio ambiente, si puede tener gran implicación.

El propio Truhaut (1977) hizo redefinición de la Ecotoxicología (reconocido como el texto pionero) donde mencionó que deben considerarse varios aspectos en términos cuantitativos dentro de una serie de procesos como los siguientes: a) Lugar, naturaleza y concentraciones relativas de la emisión contaminante.

b) Su transporte geográfico y alteraciones consecuentes a los mecanismos físicos implicados (adsorciones, absorciones, retenciones, diluciones, concentraciones, etc.).

c) Transformaciones químicas de naturaleza abiótica (hidrólisis por la humedad, oxidaciones por el aire y la luz ultravioleta, condensaciones fotoquímicas, etc.).

d) Biotransformaciones durante su transporte por los diferentes organismos en el ecosistema (biodegradación) y la bioacumulación a lo largo de las cadenas tróficas.

e) Incidencia de la exposición sobre los organismos, poblaciones o comunidades críticas o dianas.

f) Respuestas de los organismos en el tiempo.

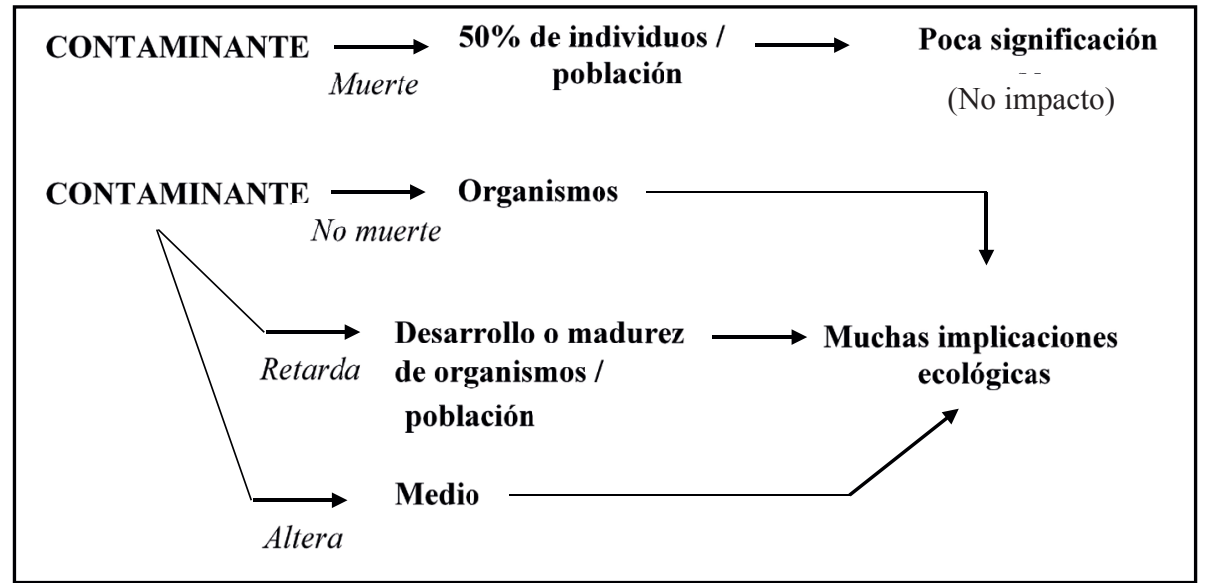

Figura 1. Interpretación del concepto sobre ecotoxicología propuesto por Truhaut (1977).

Posteriormente, Sanz (1974) mencionó que la Ecotoxicología como ciencia, estudia la polución, origen, evolución e interacciones con las moléculas que integran dinámicamente los ecosistemas, además, las acciones y efectos sobre los seres vivos que forman estos ecosistemas con su evaluación como determinantes de criteriología considerándose la profilaxis biológica o socioeconómica. En esta definición dos aspectos resultan de interés. El primero, la profilaxis en término de vigilancia; y lo segundo, la implicación que puede ocasionar la polución en el contexto social y económico.

Butler (1978), indicó que la Ecotoxicología, es la ciencia que relaciona los efectos tóxicos de los agentes químicos y físicos con los organismos vivos sobre todo en las poblaciones y las comunidades dentro de los ecosistemas bien definidos, incluyendo las transferencias de estos agentes y sus interacciones con el ambiente. En esta definición se indica que, solo los efectos tóxicos se reconocen cuando los ecosistemas se encuentran bien definidos, es decir, no obedece a cualquier espacio o hábitat geográfico. Otro aspecto significativo radicó en el dańo posible cuando existe un valor de uso en cualquier recurso secundario (factor o proceso de transferencia).

Blaise et al. (1985) plantearon que, la Ecotoxicología estudia el destino y los efectos de los contaminantes en los ecosistemas, intentando explicar las causas y prever los riesgos probables. En este sentido, se habló sobre una ecotoxicología prospectiva que evalúa la toxicidad de las sustancias antes de su producción y uso; y una ecotoxicología retrospectiva que se ocupa de confirmar, si la sustancia produce daño en el ecosistema. En esta definición, una de las ideas interesantes estuvo referida al carácter preventivo, pues lo perseguido fue entender de forma anticipada, posibles efectos adversos de los contaminantes antes de que puedan entrar en contacto con el medio ambiente. 
Por su parte, Rudolph \& Boje (1986) señalaron que la Ecotoxicología, es la ciencia que investiga los efectos de sustancias sobre los organismos. El peligro sobre las poblaciones de animales y plantas puede ser determinado mediante el uso de datos existentes tomados del ambiente (ecotoxicología retrospectiva) o mediante desarrollo de ensayos específicos (ecotoxicología prospectiva). En esta definición puede interpretarse, la necesidad de realizar mediciones y con los valores hallados, luego realizar las predicciones de su peligro. Para ello, es considerado utilizar en condiciones de laboratorio, modelos biológicos de experimentación.

En cambio, Moriarty (1988) refirió que la Ecotoxicología, es la ciencia que propone la evaluación, monitoreo, predicción del destino y efectos de xenobióticos en el ambiente. En esta definición, al parecer lo relevante estuvo en la clasificación de xenobióticos como agente que no presenta un umbral natural para su posible degradación y por tanto, la necesidad de valorar el riesgo en su manifestación.

En el caso de Pourriot \& Meybeck (1995) esbozaron que, la Ecotoxicología se basa en la combinación de los conceptos fundamentales de la ecología que apunta para entender las relaciones entre los organismos y su ambiente circundante, a través de un análisis sistémico utilizando unidades funcionales de variación con los registros experimentales de la limnología contemporánea y las propiedades físico-químicas de las sustancias antes y después que se vierten al ecosistema. Esta definición, tuvo una dimensión aplicada para condiciones de campo pero lo relevante radicó en la necesidad de valorar, cómo las fluctuaciones de parámetros determinan cambios en las estructuras y composición química donde a su vez, influyen en el comportamiento de los organismos. La influencia no necesariamente tiene que ser directa sino, puede ser mediante el medio con lo cual, sigue considerándose la propuesta inicial formulada por Truhaut. Finalmente, Tarazona \& Ramos (2014) indicaron a la Ecotoxicología como la ciencia dedicada al

Ante la interpretación teórica y usos prácticos sobre la evaluación de sustancias, compuestos, utilidad de bioensayos y generación de modelos computacionales estudio y prevención de los efectos adversos provocadas por las emisiones antropogénicas de sustancias químicas en la estructura, funciones y biodiversidad de los ecosistemas. En esta definición, puede valorarse la dinámica de cambio (enlace) que puede transcurrir en las sustancias químicas emisoras y por ende; su posible biodisponibilidad.

Probablemente por lo leído, algunos autores como Newman \& Zhao (2008) refieren que, la Ecotoxicología es una ciencia y en realidad no lo es, por cuanto carece de principios, leyes y teoría propia, ya que en ella se combina, explicaciones causales e informaciones de otras ciencias (biología, química, bioquímica, matemática, física, geología entre otras). En este saber, la Ecotoxicología es reconocida como rama sintética pudiendo ser posible que, múltiples respuestas sobre los desafíos del entorno social se manifiestan de forma compleja y donde, no se ha podido dar soluciones, ya que los datos que se incorporan siguen siendo incompletos debido al carácter aplicativo de la Ecotoxicología.

De manera general, puede definirse a la Ecotoxicología (Argota, 2016) como: Rama que se encarga en estudiar las perturbaciones estructurales y funcionales a corto, mediano y largo plazo ocasionadas por la contaminación sobre los sistemas ecológicos y donde éstos funcionan sobre la base de las acciones e interacciones entre factores abióticos y bióticos.

Al indicarse como referencia los efectos biológicos no deseados por contaminantes expuestos en el medio, éstos pueden ser diversos tales como se muestran en la figura 2 de manera que si fuera así, la continuidad sobre evaluaciones sistemáticas posibilita entender que, ciertas exposiciones influyen de manera parcial o total a la pérdida de individuos o poblaciones en los ecosistemas acuáticos.

El propósito del estudio fue indicar la ecotoxicología como herramienta predictiva sobre la evolución sostenible de los sistemas acuáticos.

referidos en diversas publicaciones de la literatura científica se seleccionó como referencia analítica al ecosistema acuático San Juan de Santiago de Cuba-Cuba (Figura 3). 


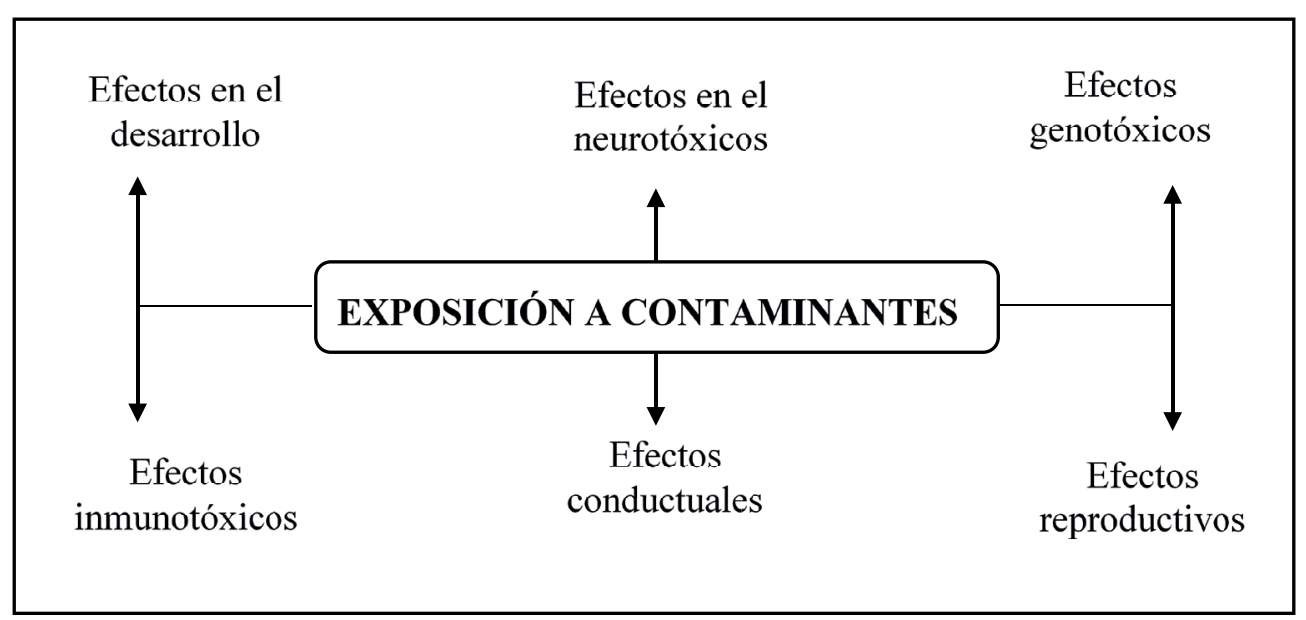

Figura 2. Efectos biológicos no deseados por la exposición a contaminantes (Argota, 2016).

\section{MATERIAL Y MÉTODOS}

Ante la interpretación teórica y usos prácticos sobre la evaluación de sustancias, compuestos, utilidad de bioensayos y generación de modelos computacionales referidos en diversas publicaciones de la literatura científica se seleccionó como referencia analítica al ecosistema acuático San Juan de Santiago de Cuba-Cuba (Figura 3).
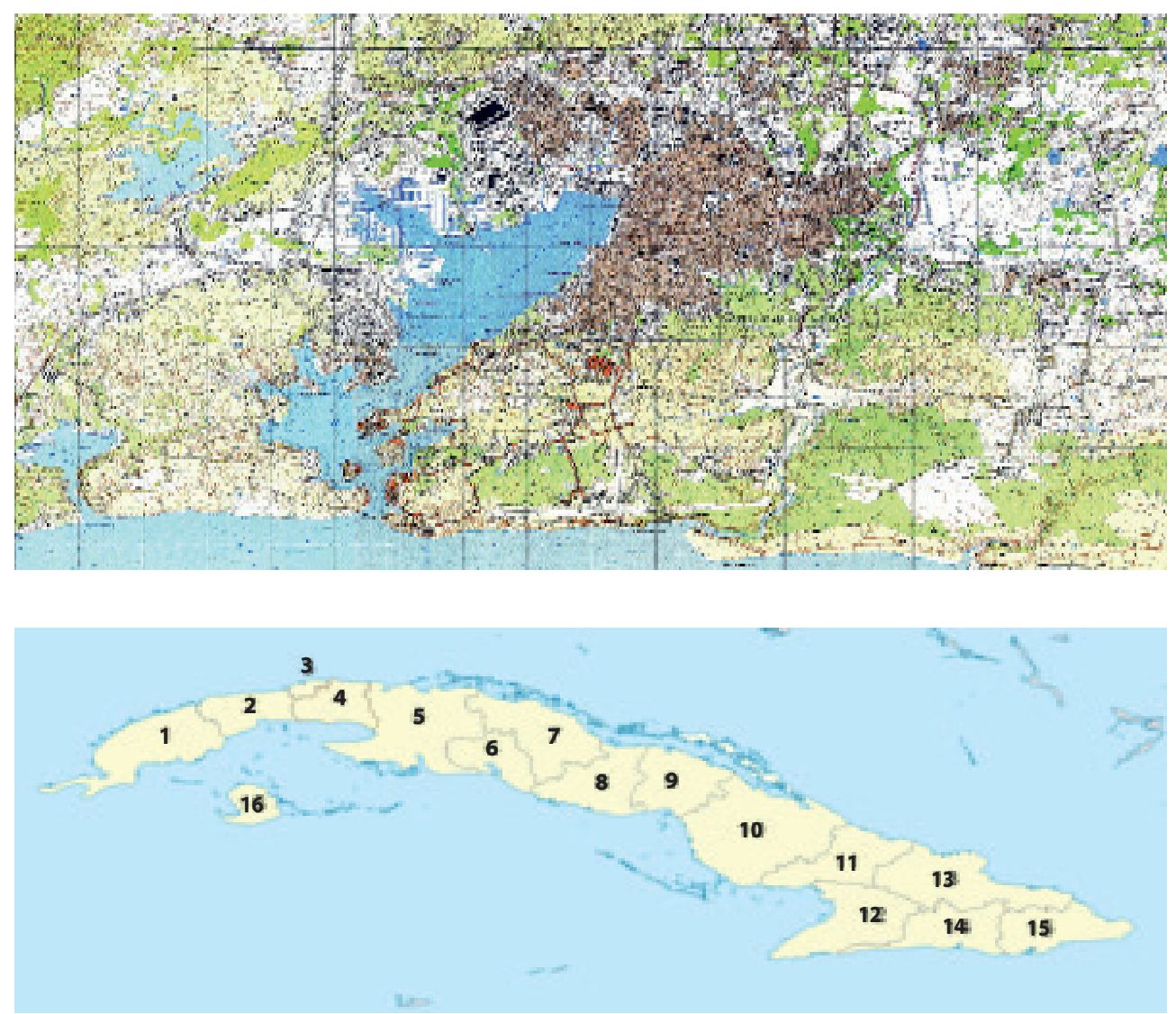

Figura 3. Ecosistema San Juan (rectángulo) en Santiago de Cuba, Cuba. 
Para determinar la calidad ambiental y evolución sostenible del ecosistema San Juan se midió durante 10 años $(2008,2013,2018)$, el factor de condición biológico (k) y la bioacumulación de metales ( $\mathrm{Pb}$ y $\mathrm{Cd}$ ) en las branquias (órganos diana) de la especie Gambusia punctata (Poey, 1854) (Poeciliidae) definida como biomonitor en ecotoxicología acuatica (Argota et al., 2013).

Se utilizó el programa estadístico profesional Statgraphics Centurion 18 para el tratamiento de los datos donde la normalidad se realizó mediante la prueba Kolmogorov-S. Para la comparación entre los años se utilizó el estadígrafo análisis de la varianza siendo la prueba de contraste múltiple de rango por Bonferroni, la que se seleccionó para establecer las diferencias entre los grupos. Se consideró significativos los resultados cuando $\mathrm{p}<0,05$.

Aspectos éticos: los autores señalan que se cumplieron todos los aspectos éticos nacionales e internacionales.

\section{RESULTADOS}

La tabla 1 muestra el factor de condición biológico $(\mathrm{K})$ en la especie biomonitor $G$. punctata durante el periodo de estudio donde hubo diferencias estadísticamente significativas entre los ańos por cada sexo (tabla 2).

Tabla 1. Factor de condición biológico / sexo / año.

\begin{tabular}{ccccc}
\hline Sexo & Indicador & 2008 & 2013 & 2018 \\
\hline machos & $\mathrm{K}$ & $0,45-0,47$ & $0,43-0,44$ & $0,37-0,41$ \\
hembras & & $0,63-0,66$ & $0,58-0,61$ & $0,55-0,58$ \\
\hline
\end{tabular}

Tabla 2. Anova (Análisis de Varianza) / contrastre múltiple de rangos / factor de condición biológico / sexo / años. $\mathrm{FV}=$ Fuentes de variación. $\mathrm{SC}=$ Suma de cuadrados. $\mathrm{gl}=$ grados de libertad. $\mathrm{CM}=$ cuadrado medio. $\mathrm{F}=$ Estadísticos de Fisher del ANOVA. $\mathrm{P}$ = probabilidad. $\mathrm{X}$ = promedio. Letras diferentes en una misma columna señalan diferencias estadísticamente diferentes $(\mathrm{P}<0,05)$.

\begin{tabular}{|c|c|c|c|c|c|}
\hline \multicolumn{6}{|c|}{ Machos } \\
\hline FV & $\mathrm{SC}$ & $\mathrm{gl}$ & $\mathrm{CM}$ & Coeficiente - F & $\mathrm{P}$ \\
\hline Entre grupos & 0,0075 & 2 & 0,0037 & 37,75 & 0,0004 \\
\hline Intra grupos & 0,0006 & 6 & 0,0001 & & \\
\hline Total (Corr.) & 0,0081 & 8 & & & \\
\hline Año & $\mathrm{X}$ & $\begin{array}{c}\text { Grupos } \\
\text { homogéneos }\end{array}$ & & & \\
\hline 2018 & 0,39 & $\mathrm{a}$ & & & \\
\hline 2013 & 0,43 & $\mathrm{~b}$ & & & \\
\hline 2008 & 0,46 & $\mathrm{~b}$ & & & \\
\hline \multicolumn{6}{|c|}{ Hembras } \\
\hline FV & SC & $\mathrm{gl}$ & $\mathrm{CM}$ & Coeficiente - F & $\mathrm{P}$ \\
\hline Entre grupos & 0,0098 & 2 & 0,0049 & 4900,00 & 0,0000 \\
\hline Intra grupos & 0,000006 & 6 & 0,000001 & & \\
\hline Total (Corr.) & 0,009806 & 8 & & & \\
\hline Año & $\mathrm{X}$ & $\begin{array}{c}\text { Grupos } \\
\text { homogéneos }\end{array}$ & & & \\
\hline 2018 & 0,56 & $\mathrm{a}$ & & & \\
\hline 2013 & 0,59 & $\mathrm{~b}$ & & & \\
\hline 2008 & 0,64 & c & & & \\
\hline
\end{tabular}

La tabla 3 muestra las concentraciones bioacumuladas de metales en las branquias de la especie biomonitor donde no hubo diferencias estadadísticamente significativas (tabla 4). 
Tabla 3. Concentración promedio de metales $\left(\mathrm{ug}^{-\mathrm{g}^{-1}}\right) /$ entre los sexos / año.

\begin{tabular}{ccccc}
\hline Sexo & Metal & 2008 & 2013 & 2018 \\
\hline Macho-Hembras & $\mathrm{Pb}$ & $0,04 \pm 0,01$ & $0,03 \pm 0,02$ & $0,04 \pm 0,02$ \\
& $\mathrm{Cd}$ & $16,44 \pm 0,017$ & $16,77 \pm 0,011$ & $16,78 \pm 0,008$ \\
\hline
\end{tabular}

Tabla 4. Anova (Análisis de Varianza) / bioacumulación de metales / sexo. FV = Fuentes de variación. SC = Suma de cuadrados. $\mathrm{gl}=$ grados de libertad $. \mathrm{CM}=$ cuadrado medio. $\mathrm{F}=$ Estadísticos de Fisher del ANOVA. $\mathrm{P}=$ probabilidad .

\begin{tabular}{lccccc}
\hline \multicolumn{1}{c}{ FV } & $\mathrm{SC}$ & $\mathrm{gl}$ & $\mathrm{CM}$ & Coeficiente $-\mathrm{F}$ & $\mathrm{P}$ \\
Entre grupos & 0,0002 & 2 & 0,0001 & 1,00 & 0,42 \\
Intra grupos & 0,0006 & 6 & 0,0001 & & \\
Total (Corr.) & 0,0008 & 8 & & & \\
& & & $\mathrm{Cd}$ & & \\
\multicolumn{1}{c}{$\mathrm{FV}$} & $\mathrm{SC}$ & $\mathrm{gl}$ & $\mathrm{CM}$ & Coeficiente $-\mathrm{F}$ & $\mathrm{P}$ \\
Entre grupos & 0,222289 & 2 & 0,111144 & 3,51 & 0,09 \\
Intra grupos & 0,189867 & 6 & 0,0316444 & & \\
Total (Corr.) & 0,412156 & 8 & & & \\
\hline
\end{tabular}

\section{DISCUSIÓN}

La predicción del riesgo ambiental sobre los ecosistemas acuáticos desde el punto de vista ecotoxicológico ha sido medida mediante modelos informatizados (Chapman \& Riddle, 2003; Patlewicz \& Fitzpatrick, 2016), uso de índices (López et al., 2016) y bioensayos (Fahd et al., 2014; Sadeghi \& Hedayati, 2014; Malaj et al., 2016). Sin embargo, estas mediciones de forma independientes, quizás limiten algunas interpretaciones sobre la calidad ambiental sostenible de los recursos acuáticos.

En ecotoxicología acuática, el uso de organismos naturales como los peces, permiten evaluar la calidad histórica de los sistemas (Heidary et al., 2012; Hamza, 2014; Çiftçi et al., 2015) siendo relevante, si esta aplicación se basa en condiciones de biomonitoreo (AbdAllah, 2017), ya que los peces posibilitan estimar, el impacto y consecuencia de la exposición (Baptista et al., 2013; Marcato et al., 2014; Bianchi et al., 2015; Adeogun et al., 2016; Alegre et al., 2018; Tomailla \& Iannacone, 2018). Cualquier cambio biológico expresado a cualquier nivel señala, posible alteración ocasionada por actividades antropogénicas. Cada nivel de respuesta, es un indicador del riesgo ecotoxicológico al que está siendo expuesta, alguna población (Manrique et al., 2013).

Argota \& Iannacone (2017) indicaron que en biomonitores pueden determinarse respuestas bioquímicas, fisiológicas, morfológicas, conductuales, inmunológicas entre otras; pero lo significativo para medir el riesgo de perturbación ambiental deberá usarse de forma permanente el(los) biomarcador(es) más sensible(s) como criterio fidedigno de monitoreo sobre el peligro expresado por el contaminante expuesto. En este sentido, el uso de cualquier respuesta, constituye un biomarcador de información cualitativa y cuantitativa para describir la exposición.

En este estudio, uno de los biomarcadores de efecto en peces que se utilizó fue el factor $\mathrm{K}$, el cual relaciona la longitud y el peso (Cifuentes et al., 2012), donde se permite cierta medida del crecimiento y estado de nutrición de los peces. Cuando existe poca disponibilidad alimentaria, puede evidenciarse una declinación paulatina en el factor $\mathrm{K}$ siendo de igual modo referido por Felipa et al. (2016). El factor K fue disminuyendo desde el 2008 hasta el 2018 expresando perturbaciones sobre la calidad ambiental del ecosistema San Juan. Ante las perturbaciones ambientales de los ecosistemas acuáticos, las branquias de los peces son sitios principales de expresión (Van der Oost et al., 2003) y su evaluación temprana representa una imagen integrada y ecológicamente significativa del impacto de contaminantes como la exposición biodisponible a metales en el tiempo (Jonge et al., 2015).

En el estudio, no hubo diferencias significativas $(\mathrm{p}<0,05)$ entre las concentraciones promedio (estimada entre 
machos y hembras) de $\mathrm{Pb}$ y $\mathrm{Cd}$ desde el 2008 hasta el 2018 lo que evidenció, persistencia y estado biodisponible ambiental. Cuando los metales se encuentran en este estado pueden ocasionar dańos irreversibles y afectar determinada respuesta biológica.

Los biomarcadores señalaron que, existió limitada estabilidad ambiental en el tiempo y por ende; poca sostenibilidad como recurso ecosistémico para el San Juan donde cada análisis se basó en los preceptos de la ecotoxicología.

\section{REFERENCIAS BIBLIOGRÁFICAS}

AbdAllah, A.T. 2017. Efficiency of invertebrate animals for risk assessment and biomonitoring of hazardous contaminants in aquatic ecosystem, a review and status report. Journal of Environmental Risk Assessment and Remediation, 1: 22-24.

Adeogun, A.O.; Ibor, O.R.; Onoja, A.B. \& Arukwe, A. 2016. Fish condition factor, peroxisome proliferator activated receptors and biotransformation responses in Sarotherodon melanotheron from a contaminated freshwater dam (Awba Dam) in Ibadan, Nigeria. Marine Environmental Research, 121: 74-86.

Alegre, A.; Bonifaz, E.; Lee, S.E.S.; Alvariño, L. \& José Iannacone. 2018. Monitoreo ecotoxicológico de una Cuenca en Huancavélica, Perú afectada por metales pesados. - Revista electrónica de Veterinaria, 19: 1-14 http://www.veterinaria. org/revistas/redvet/n050518.html

Argota, P.G. \& Iannacone, J. 2017. Predicción cuantitativa mediante biomarcadores de uso permanente como nuevo criterio para biomonitores en ecotoxicología acuática. The Biologist (Lima), 17: $141-153$.

Argota, P.G. 2016. Monitoreo biológico ambiental en ecotoxicología. KOPYgraf Ed. Cusco.

Argota, P.G.; Iannacone, J. \& Fimia, D.R. 2013. Características de Gambusia punctata (Poeciliidae) para su selección como biomonitor en ecotoxicología acuática en Cuba. The Biologist (Lima), 11: 229-236.

Baptista, J.; Pato, P.; Duarte, A.C. \& Pardal, M.A. 2013. Organochlorine contaminants in different tissues from Platichthys flesus (Pisces, Pleuronectidea). Chemosphere, 93: 1632-1638.

Bianchi, E.; Goldoni, A.; Trintinaglia, L.; Lessing, G.; Silva, C.E.M.; Nascimento, C.A.; Ziulkoski, A.L.; Spilki, F.R. \& Silva, L.B. 2015. Evaluation of genotoxicity and cytotoxicity of water samples from the Sinos River Basin, southern Brazil. Brazilian. Brazilian Journal of Biology, 75: $68-74$.

Blaise, C.; Berminghan, N. \& Van Coillie, R. 1985. The integrated ecotoxicological approach to assessment of ecotoxicity. Water quality bulletin, 10: 3-7, 60-61.

Butler, G.C. 1978. Principles of Ecotoxicology. SCOPE 12, John Wiley and Sons, New York. pp. 372.

Carter, J. \& Howe, J. 2006. The water framework directive and the strategic environmental assessment directive: exploring the linkages. Environmental Impact Assessment Review, 26: 287-300.

Chapman, P.M. \& Riddle, M.J. 2003. Missing and needed: polar marine ecotoxicology. Marine Pollution Bulletin, 46: 927-928.

Çiftçi, N.; Ay, Ö.; Karayakar, F.; Cicik, B. \& Erdem, C. 2015. Effects of zinc and cadmium on condition factor, hepatosomatic and gonadosomatic index of Oreochromis niloticus. Fresenius Environmental Bulletin, 24: 3871-3874.

Cifuentes, R.; González, J.; Montoya, G.; Alfonso, J.; Ortiz, N.; Piedra, P. \& Habit, E. 2012. Relación longitud-peso y factor de condición de los peces nativos del río San Pedro (cuenca del río Valdivia, Chile). Gayana (Concepción), 75: 101-110.

Destrieux, D.; Laurent, F.; Budzinski, H.; Pedelucq, J.; Vervier, P. \& Gerino, M. 2017. Drug residues in urban water: A database for ecotoxicological risk management. Science of the Total Environment, 609: 927-941.

Fahd, F.; Khan, F.; Hawboldt, K. \& Abbassi, R. 2014. Developing a novel methodology for ecological risk assessment of thiosalts. Stochastic Environmental Research and Risk Assessment, 28: 383-391.

Felipa, G.; Blas, W. \& Alcántara, F. 2016. Relación longitud-peso, factor de condición y tabla 
estándar del peso de mil alevinos de gamitana (Colossoma macropomum, Cuvier, 1818) criados en estanques artificiales. Folia Amazónica, 25: $17-24$.

Forbes, V.E. \& Galic, N. 2016. Next-generation ecological risk assessment: predicting risk from molecular initiation to ecosystem service delivery. Environment International, 91: 215219.

Hamza, C.A. 2014. Usefulness of bioindicators and biomarkers in pollution biomonitoring. International Journal of Biotechnology for Wellness Industries, 3: 19-26.

Heidary, S.; Imanpour-Namin, J. \& Monsefrad, F. 2012. Bioaccumulation of heavy metals $\mathrm{Cu}, \mathrm{Zn}$, and $\mathrm{Hg}$ in muscles and liver of the stellate sturgeon (Acipenser stellatus) in the Caspian Sea and their correlation with growth parameters. Iranian Journal of Fisheries Sciences, 11: 325-337.

Hommen, U.; Baveco, J.M.; Galic, N. \& van den Brink, P.J. 2010. Potential application of ecological models in the European environmental risk assessment of chemicals I: review of protection goals in the EU directives and regulations. Integrated Environmental Assessment and Management, 6: 325-337.

Hughes, S.R.; Kay, P. \& Brown, L.E. 2013. Global synthesis and critical evaluation of pharmaceutical data sets collected from river systems. Environmental Science \& Technology, 47: 661-677.

Jonge, M.; Belpaire, C.; Van Thuyne, G.; Breine, J. \& Bervoets, L. 2015. Temporal distribution of accumulated metal mixtures in two feral fish species and the relation with condition metrics and community structure. Environmental Pollution, 197: 43-54.

Lam, P.K.S. \& Gray, J.S. 2003. The use of biomarkers in environmental monitoring programmes. Marine Pollution Bulletin, 46: 182-186.

López, I.D.; Figueroa, A. \& Corrales, J.C. 2016. A systematic mapping of water quality prediction using computational intelligence techniques. Revista Ingenierías Universidad de Medellín, 15: 35-51.
Lopez, S.R.; Jurado, A.; Vazquez, S.E.; Carrera, J.; Petrovic, M. \& Barcelo, D. 2013. Occurrence of 95 pharmaceuticals and transformation products in urban groundwaters underlying the metropolis of Barcelona, Spain. Environmental Pollution, 174: 305-315.

Malaj, E.; Guenard, G.; Schafer, R.B. \& von der Ohe, P.C. 2016. Evolutionary patterns and physicochemical properties explain macroinvertebrate sensitivity to heavy metals. Ecological Applications, 26: 1249-1259.

Manrique, W.G.; Figueiredo, M.A.P. \& Machado, N.J.G. 2013. Dissipation and environmental risk of fipronil on aquatic environment. The Biologist (Lima), 11: 107-117.

Marcato, A.C.C.; Yabuki, A.T. \& Fontanetti, C.S. 2014. Nickel exposure promotes osmoregulatory disturbances in Oreochromis niloticus gills: histopathological and energy dispersive spectrometry analysis. Environmental Science and Pollution Research, 21: 13095-13102.

Moriarty, F. 1988. Ecotoxicology. The study of pollutants in ecosystems. $2^{\text {da }}$ Ed. Academic Press, Gran Bretaña. pp 289.

Newman, M.C. \& Zhao, Y. 2008. Ecotoxicology nomenclature: $L C, L D, L O C, L O E C, M A C$. In: Jorgensen, S.E. \& Fath, B. (Eds.). Encyclopedia of Ecology; Elsevier. pp. 1187-1193.

O’Brien, A.L. \& Keough, M.J. 2014. Ecological responses to contamination: a meta-analysis of experimental marine studies. Environmental Pollution, 195: 185-191.

Pal, A.; He, Y.; Jekel, M.; Reinhard, M. \& Gin, K.Y.H. 2014. Emerging contaminants of public health significance as water quality indicator compounds in the urban water cycle. Environment International, 71: 46-62.

Patlewicz, G. \& Fitzpatrick, J.M. 2016. Current and future perspectives on the development, evaluation, and application of in silico approaches for predicting toxicity. Chemical Research in Toxicology, 29: 438-451.

Pedersen, S.; Palmqvist, A.; Thorbek, P.; Hamer, M. \& Forbes, V. 2013. Pairing behavior and 
reproduction in Hyalella azteca as sensitive endpoints for detecting long-term consequences of pesticide pulses. Aquatic Toxicology, 144: 59-65.

Pourriot, R. \& Meybeck, M. 1995. Limnologie Générale. Masson, Paris, Milan, Barcelone.

Rudolph, P. \& Boje, R. 1986. Okotoxikologie. Grundlage für die okotoxikologische Bewertung von Umweltchemikaliem nach dem Chemikaliengesetz, ecomed.

Sadeghi, A. \& Hedayati, A. 2014. Investigation of $\mathrm{LC}_{50}$, NOEC and LOEC of Glyphosate, Deltamethrin and Pretilachlor in Guppies (Poecilia reticulata). Iranian Journal of Toxicology, 8: 1124-1129.

Sanz, S.F. 1974. Sentido y Posibilidades de la Toxicología Ambiental. Discurso de Apertura del Curso Académico 1974-75. U.C.M.

Simpson, S.L., Batley, G.B. \& Chariton, A.A. 2013. Revision of the ANZECC/ARMCANZ Sediment Quality Guidelines. CSIRO Land and Water Science.

Stark, J.D.; Vargas, R. \& Banks, J.E. 2007. Incorporating ecologically relevant measures of pesticide effect for estimating the compatibility of pesticides and biocontrol agents. Journal of Economic Entomology, 100: 1027-1032.

Tarazona, J.V. \& Ramos, P.M.J. 2014. Ecotoxicology. Enciclopedia of Toxicology ( ${ }^{\text {rd }}$ Ed.) pp. 276280.
Tomailla, J. \& Iannacone, J. 2018. Toxicidad letal y subletal del arsénico, cadmio, mercurio y plomo sobre el pez Parachaeirodon innesi neon tetra (Characidae). Revista de Toxicologia, 35: 95105.

Truhaut, R. 1977. Eco-toxicology-objectives, principles and perspectives. Ecotoxicology and Environmental Safety, 1: 151-173.

USEPA (United States Environmental Protection Agency). 2015. Water Quality Standards Handbook. Chapter 7: Water Quality standards and the water quality-based approach to Pollution Control. Office of Water, Washington, DC.

Van der Oost, R.; Beyer, J. \& Vermeulen, N.P.E. 2003. Fish bioaccumulation and biomarkers in environmental risk assessment: a review. Environmental Toxicology and Pharmacology, 13: $57-149$.

Weber, F.A., aus der Beek, T.; Bergmann, A.; Carius, A.; Grüttner, G.; Hickmann, S.; Ebert, I.; Hein, A.; Küster, A.; Rose, J.; Koch-Jugl, J. \& Stolzenberg, H.C. 2014. Les produits pharmaceutiques dans l'environnement-perspective à l'échelle mondiale. Présence, effets, et action concertée potentielle au titre de la SAICM. Umwelt Bundesamt.

Received December 2, 2018.

Accepted December 28, 2018. 